\title{
TV/Series
}

$11 \mid 2017$

Philosopher avec Battlestar Galactica

\section{La machine comme " prothèse d'origine ": réflexion philosophique sur le sujet humain dans Battlestar Galactica de Ronald D. Moore}

\section{René Lemieux}

\section{(2) OpenEdition}

Journals

Édition électronique

URL : http://journals.openedition.org/tvseries/1981

DOI : 10.4000/tvseries.1981

ISSN : 2266-0909

Éditeur

GRIC - Groupe de recherche Identités et Cultures

Référence électronique

René Lemieux, «La machine comme « prothèse d'origine » : réflexion philosophique sur le sujet humain dans Battlestar Galactica de Ronald D. Moore », TV/Series [En ligne], 11 | 2017, mis en ligne le

11 juin 2017, consulté le 03 mai 2019. URL : http://journals.openedition.org/tvseries/1981 ; DOI :

10.4000/tvseries.1981

Ce document a été généré automatiquement le 3 mai 2019.

\section{(c) (i) (9)}

TV/Series est mis à disposition selon les termes de la licence Creative Commons Attribution - Pas d'Utilisation Commerciale - Pas de Modification 4.0 International. 


\title{
La machine comme « prothèse d'origine " : réflexion philosophique sur le sujet humain dans Battlestar Galactica de Ronald D. Moore
}

\author{
René Lemieux
}

1 Battlestar Galactica de Ronald D. Moore est une série télévisée diffusée de 2003 à 2009. Il s'agit d'une reprise réimaginée - un reboot - d'une première série (de Glen Larson) diffusée en 1978. L'aspect formel du reboot n'est pas anodin. Comme l'explique Mehdi Achouche, reboot provient du langage informatique et signifie qu' « on efface [...] la mémoire vive pour tout redémarrer $»^{1}$. Le terme est ainsi devenu un moyen, dans le discours ordinaire et en particulier dans le discours politique, pour parler d'un retour à l'origine: «Recommencer à zéro, effacer une partie de l'histoire nationale pour emprunter une nouvelle voie chronologique et mieux se développer ${ }^{2}$. " Battlestar Galactica peut aussi se voir comme une réflexion sur la « République » comme forme politique. La «destinée manifeste » que certains ont vue dans Battlestar Galactica serait en quelque sorte un "Texte fondateur", comme l'appelait Michel Freitag, à la manière de la constitution américaine à laquelle tout enjeu politique revient toujours ${ }^{3}$. Une telle critique permet de sérieusement remettre en question l'universalité humaine à laquelle prétend la série ${ }^{4}$. Mon propos sera toutefois différent : à mon sens, le «texte premier» auquel peut faire référence la série est l'enjeu du rapport Homme-Machine. Il s'agit ainsi moins de comprendre à quoi pensaient les créateurs de la série, que de penser à partir d'elle. À cet égard, je comprends le «programme» qu'est l'origine humaine comme incluant un «improgrammable», la Machine, pensée ici comme une "prothèse d'origine », une extériorité secondaire, mais essentielle pour penser l'intériorité originelle ${ }^{5}$.

2 Battlestar Galactica a fait l'objet de nombreux commentaires sur le rôle et la portée politique de l'imaginaire de la Machine qu'elle propose. Réalisée dans une Amérique encore aux prises avec les peurs liées au terrorisme, Battlestar Galactica offre, avec les 
Cylons (des robots humanoïdes, principaux antagonistes de la série), une image de l'Autre dont les frontières s'effacent petit à petit avec la progression des épisodes. Au lendemain des attaques du 11-septembre, un imaginaire de l'ennemi intérieur s'est formé avec la peur des musulmans. Battlestar Galactica reprend, en la transformant, la "guerre contre le terrorisme » (War on Terror) 6 . À l'imaginaire de l'ennemi politique déshumanisé, la série associe aussi le catastrophisme de la science-fiction contemporaine ${ }^{7}$ en y ajoutant une autre peur : comme pour 2001: A Space Odyssey (Stanley Kubrick, 1968), Terminator (James Cameron, 1984) ou encore The Matrix (Wachowskis, 1999), cette nouvelle crainte est de voir les Machines se révolter contre leurs créateurs. Tous ces éléments font de Battlestar Galactica un concentré des peurs de notre époque. Pourtant, parce que l'épisode final de la série vient entièrement remettre en question ce qui nous était donné au point de départ, Battlestar Galactica se transforme en questionnement philosophique complexe.

3 L'objectif de cet article sera d'abord de questionner le préjugé de notre époque, très « moderne » à cet égard, du danger d'une trop grande technicisation de notre monde, de la place trop grande que prend la «Machine» ou la robotique, ou encore l'intelligence artificielle vue comme une compétitrice à la place de l'Homme dans le monde. Je ne définis pas plus à présent ces deux mots de "Machine » et d'« Homme", car l'un des objectifs de l'article sera de montrer qu'une série télévisée comme Battlestar Galactica peut réussir à redéfinir nos idées préconçues. La réflexion sur la « nature » de l'homme que je voudrais entamer se basera sur l'idée que Machine et Homme doivent se penser de pair : l'Homme, pour pouvoir être conçu, nécessite la Machine.

4 Afin de présenter cette hypothèse sur les rapports Homme-Machine dans Battlestar Galactica, j'expliquerai d'abord une des pensées les plus originales contre l'imaginaire du danger, celle d'Alan Turing, créateur de la machine «ordinateur ». J'exposerai dans un premier temps certains éléments de sa pensée qui pourrait se résumer à penser l'esprit humain comme d'abord mécanique. Dans un deuxième temps, je présenterai quelques éléments du conflit entre Homme et Machine avec quelques exemples de fiction. Je m'intéresserai particulièrement à Star Trek (le premier film, réalisé par Robert Wise, 1979) et Blade Runner (Ridley Scott, 1982) qui me semblent les plus intéressants dans le domaine de l'idéologie sur la Machine. Je terminerai avec le cas de Battlestar Galactica et particulièrement du dernier épisode qui renverse plusieurs préjugés sur la relation entre Homme et Machine. Je tenterai à cet égard de montrer que c'est la forme «télésérie » ou «feuilleton télévisé » qui permet une prise de conscience sur cette relation qui pourrait se résumer ainsi : La pensée mécanique, c'est faire une distinction binaire entre l'Homme et la Machine; penser humainement, c'est accepter l'événement de la Machine. Pour ce faire, je mobiliserai principalement Jacques Derrida qui, dans Monolinguisme de l'autre, qualifie la langue de "prothèse d'origine ». L'intérêt ici est de comprendre le langage comme ce qui fonctionne dans une répétition (du même) et à la fois fonctionne nécessairement dans la différence.

\section{Le lieu de la machine et son imaginaire catastrophique}

Le « problème » de la place de la Machine a été récemment réactualisé dans un entretien donné à la $\mathrm{BBC}$ par le physicien Stephen Hawking, pour qui l'intelligence artificielle est le plus grand danger que court l'humanité aujourd'hui ${ }^{8}$. Ce problème semble au cœur de la Modernité qui n'aurait pas su se modérer et serait perpétuellement dans une hubris autodestructrice. Par Modernité, j'entends le moment fondateur de la tabula rasa en 
histoire de la philosophie et l'institution du sujet (humain) pleinement conscient de manière réflexive de lui-même. Ce moment fondateur est notamment conceptualisé par René Descartes dans Le Discours de la méthode qui offre un double discours d'exclusion, de l'animal et de la machine :

Et je m'étois ici particulièrement arrêté à faire voir que s'il y avoit de telles machines qui eussent les organes et la figure extérieure d'un singe ou de quelque autre animal sans raison, nous n'aurions aucun moyen pour reconnoître qu'elles ne seroient pas en tout de même nature que ces animaux ; au lieu que s'il y en avoit qui eussent la ressemblance de nos corps, et imitassent autant nos actions que moralement il seroit possible, nous aurions toujours deux moyens très certains pour reconnoître qu'elles ne seroient point pour cela de vrais hommes : dont le premier est que jamais elles ne pourroient user de paroles ni d'autres signes en les composant, comme nous faisons pour déclarer aux autres nos pensées. [...] Et le second est que, bien qu'elles fissent plusieurs choses aussi bien ou peut-être mieux qu'aucun de nous, elles manqueroient infailliblement en quelques autres, par lesquelles on découvriroit qu'elles n'agiroient pas par connoissance, mais seulement par la disposition de leurs organes ${ }^{9}$.

6 C'est d'abord le langage (raisonné) qui peut distinguer l'homme des animaux qui ne sont en définitive que des machines sans âme. S'il y a des animaux qui peuvent parler (comme les pies et les perroquets), ajoute Descartes, ils ne le font que mécaniquement, c'est-à-dire par répétition du même. L'imaginaire de la Machine dans la modernité devient en quelque sorte l'envers de ce par quoi sera constituée l'humanité. Pour reprendre une suite de qualifications exposées par Jacques Derrida, on pourrait voir du côté de la machine comme chose inorganique et répétitive, qu'elle
serait destinée à reproduire impassiblement, insensiblement, sans organe ni organicité, l'ordre reçu. En état d'anesthésie, elle obéirait ou commanderait sans affect ni auto-affection, en automate indifférent, à un programme calculable. Son fonctionnement, sinon sa production, n'aurait besoin de personne. Puis il est difficile de concevoir un dispositif purement machinal sans quelque matière inorganique.
Dison bien inorganique. Inorganique, autrement dit non vivant, parfois mort, mais toujours, en principe, insensible et inanimé, sans désir, sans intention, sans spontanéité. L'automaticité de la machine inorganique n'est pas la spontanéité qu'on prête au vivant organique ${ }^{10}$.

Or, la pensée contemporaine et l'imaginaire qui lui est conjoint s'instituent dans le danger de voir la Machine prendre le contrôle. La machine serait alors organique, mais par imitation: elle imite le vivant, le répète, le feint, et par cela se parjure dans cette contrefaçon. C'est une énième reprise du mythe de Frankenstein où la créature se rebelle contre son créateur. Une série télévisée comme Battlestar Galactica, bien qu'elle suive en tout point cet imaginaire, me semble toutefois proposer une remise en question justement sur ce point précis de l'exclusion: avec Battlestar Galactica, le machinique comme répétition est incorporé dans ce qui fait que l'Homme est humain.

On peut situer l'origine de la peur de l'intelligence artificielle dans la fiction avec 2001: A Space Odyssey de Stanley Kubrick (1968) où une équipe d'astronautes est confrontée à une intelligence artificielle devenue autonome et consciente d'elle-même. Dénommée HAL $9000^{11}$, l'intelligence robotique tente de prendre le contrôle du vaisseau spatial et de se débarrasser, un à un, des membres de l'équipage. L'image de l'intelligence artificielle 
ici donnée est celle d'un être maléfique encore très «humain", mais sans émotion: rigide, logique, insensible, l'intelligence artificielle se rapproche de l'humain, mais ne s'y identifie pas complètement ${ }^{12}$.

Une dizaine d'années plus tard, en 1979, le premier film de la série Star Trek ${ }^{13}$ donne un nouvel exemple de catastrophisme à propos de la machine. On y raconte l'histoire d'une entité mystérieuse se nommant V'Ger, qui détruit tout sur son passage. Cette destruction - on le découvre au cours du film - est la désintégration des réalités physiques que cet " organisme" rencontre, transformées (ou traduites) en code, donc en information. Lorsqu'elle rencontre une créature vivante, V'Ger la transpose en code "équivalent " (l'ADN), l'ingère, et peut par la suite la reproduire mécaniquement en la répliquant. C'est ce qui arrivera à l'un des personnages, Ilia, une femme extraterrestre (jouée par Persis Khambatta), happée par ce qui se dévoilera comme une machine, qui la reproduira « en chair " (à partir de son code ADN), pour servir de relais communicationnel avec les membres de l'équipage et ainsi exprimer ses demandes. Détruisant tout sur son passage, V'Ger s'arrêtera juste avant d'atteindre la Terre et enverra un message codé pour son " créateur ». Après de multiples enquêtes et conjonctures sur ce que signifie "créateur " et tentatives de déchiffrer le code qui lui est destiné, l'équipage découvrira la vérité sur V'Ger: se rendant dans l'antre de l'organisme, l'équipage s'aperçoit que ce nom est une déformation de Voyager, et plus précisément de Voyager VI, une sonde (fictive ${ }^{14}$ ) envoyée de la Terre au $\mathrm{xx}^{\mathrm{e}}$ siècle avec pour objectif de recueillir le plus d'informations possibles sur l'univers, pour ensuite revenir vers la Terre, à ses "créateurs", c'est-à-dire les programmeurs originaux de la NASA. Le code envoyé n'était qu'un simple signal radio que le monde futuriste fictif de Star Trek ne savait plus reconnaître. Selon ce qu'on apprend dans le film, une fois arrivé au bout de l'univers, Voyager VI a rencontré une civilisation composée d'êtres mi-organiques mi-mécaniques qui ont pris la sonde pour un des leurs, l'ont améliorée et l'ont renvoyée vers la Terre pour accomplir son programme. Le passage le plus pertinent pour mon propos provient d'une des dernières scènes où les membres de l'équipage, présents sur V'Ger, cherchent à comprendre la nature de cet organisme :

SPOCK. Captain, V'Ger is a child. I suggest you treat it as such.

KIRK. A child?

SPOCK. Yes, captain, a child. Evolving, learning, searching, instinctively needing.

DECKER. Needing what?

MCcoY. Spock! This thing is about to wipe out every living thing on Earth. Now what do you suggest we do? Spank it?

SPOCK. It only knows that it needs, Commander. But like so many of us, it does not know what.

10 Se trouve là résumé tout le problème du rapport Homme-Machine tel que le pense la philosophie occidentale. Or, ce rapport est en continuité avec d'autres préjugés exprimés à travers le temps. L'Homme comme concept, si on le définit à partir de la machine, a aussi été historiquement défini à partir d'autres altérités, notamment celles de la femme, de l'enfant, et de l'animal. Dans l'exemple de Star Trek, V'Ger est à la fois machine, femme et enfant, et doit être traitée « comme telle ». Être une machine - une femme, un enfant dans ce cas, c'est être moins qu'un homme, c'est souffrir d'un manque, celui de ne pas être arrivé à maturité ${ }^{15}$. Il est intéressant de constater que la manifestation de ce manque se présente par le langage. La caractéristique machinique (ou féminine, ou enfantine) de V'Ger, c'est de prendre tout au pied de la lettre. V'Ger ne comprend pas la métaphore, elle n'a pas d'« esprit " - on pourrait ajouter: elle ne comprend pas l'humour ${ }^{16}$. Elle suit strictement son programme sans le questionner et, le suivant ainsi, elle met tout en 
danger - mais de manière innocente. A rebours du scénario de 2001: A Space Odyssey, la force destructrice de V'Ger provient de son impuissance à imiter intégralement l'humanité ${ }^{17}$.

11 D'autres films interrogeront la relation Homme-Machine plus radicalement, jusqu'à questionner l'essence de l'Homme ou ce qui fait qu'un Homme est un Homme. Blade Runner (Ridley Scott, 1982) est un de ces films. Adaptation d'un roman de Philip K. Dick, Do Androids Dream of Electric Sheep ${ }^{18}$ ?, Blade Runner raconte l'histoire d'un détective, appelé «blade runner ", engagé pour enquêter sur la présence de "réplicants » sur Terre, des robots ayant forme humaine et capables d'éprouver des sentiments - modèles de robots interdits sur Terre depuis leur révolte peu de temps avant le début du récit. On apprend au début du film que la compagnie qui fabrique les réplicants en a développé un nouveau modèle dont les émotions sont plus complexes, mais surtout - et cela constitue une différence radicale avec les anciens modèles -, le « modèle 6 » n'a pas la conscience d'être un robot. Le robot ne possède donc pas une faculté supplémentaire lui permettant d'être " conscient de lui-même ", mais il possède en quelque sorte un manque originaire qu'il faut suppléer par une mémoire artificielle. Cette caractéristique est à l'inverse de plusieurs récits où la machine devient dangereuse à cause d'un supplément de conscience ${ }^{19}$. D'où le développement, dans ce film, d'une question très dickienne : un robot qui ne sait pas qu'il est un robot est-il toujours un robot? En effet, un des éléments de la "personnalité » de ces robots est la mémoire qu'on leur a implantée lors de leur fabrication. Leur mémoire récente, créée à partir de la mémoire d'un individu humain, n'a pas, pour eux, de caractère artificiel.

Or, dans Blade Runner - et c'est une des seules fictions à oser cela -, le personnage antagoniste, le réplicant nommé Roy Batty démontre son " humanité » à la toute fin du film, par un geste miséricordieux envers le blade runner à sa poursuite, le sauvant d'une mort assurée et acceptant, du coup, sa propre mortalité et sa mort inéluctable ${ }^{20}$. L'inverse est simultanément vrai : alors que le récit se développe, le blade runner Rick Deckard (dont le nom joue avec « René Descartes »), incarné par Harrison Ford, dévoile ses souvenirs et un rêve récurrent à propos d'une licorne. La scène finale présente ce blade runner partant pour une nouvelle vie avec Rachel (Sean Young); l'on aperçoit une petite licorne de papier (pliage du type origami) fabriquée par un des personnages secondaires (Gaff, joué par Edward James Olmos), ce qui suggère que Deckard serait lui-même un réplicant dont Gaff connaîtrait les rêves les plus secrets. Fait intéressant, cette interprétation n'est pas admise par tous les collaborateurs au film, comme si l'on admettait aisément qu'un réplicant puisse dévoiler son humanité, mais qu'il était bien plus difficile d'admettre que l'homme puisse dévoiler sa machinalité.

Le retournement du film Blade Runner consiste bien à montrer que l'assurance de notre humanité n'est jamais inentamée. Battlestar Galactica présente aussi la possibilité que ce qu'on conçoit comme l'humanité ne soit jamais «pur» ou intact: l'enjeu du questionnement ne provient pas d'une humanité en supplément à la machinalité, mais d'une machinalité entamant toujours-déjà l'humanité. Ce n'est plus la machine qui tente d'imiter l'homme, mais l'homme qui ne peut pas s'empêcher d'imiter la machine. Afin d'explorer cette idée, je me propose de revoir rapidement une des réflexions les plus originales sur l'intelligence artificielle : celle d'Alan Turing. 


\section{Les pelures d'oignon de l'esprit humain}

Mathématicien britannique né en 1912 et mort en 1954, Alan Turing peut être considéré comme le premier véritable penseur de l'intelligence artificielle et de la machine comme pensée $^{21}$. Dans son article "Computing Machinery and Intelligence ${ }^{22}$ ", il remet en question toutes les tentatives de critique de l'ordinateur comme conscience. L'article de Turing est devenu célèbre pour son fameux test, dit « test de Turing » ou encore « jeu de l'imitation ", qui se constitue d'abord comme une expérience de pensée avant de devenir un véritable test. Dans son article, Turing invente une situation afin de répondre à la question: «Est-ce que les machines pensent?» C'est donc d'abord un exercice "pédagogique ». Or, puisque "penser» demeure un terme ambigu, Turing propose de reformuler la question en «Est-ce que les machines peuvent accomplir ce que nous (à titre d'entités pensantes) pouvons faire? » La situation hypothétique se présente d'abord comme suit : imaginons trois personnes (nommées A, B et C). A et B sont dans une salle fermée, $C$ est dans une autre salle. A et $B$ peuvent communiquer avec $C$, mais seulement sous forme de transcription (donc sans la voix ou les gestes). A est un homme et B une femme, et chacune des deux personnes tentera de convaincre $C$ (qui ne peut ni les entendre ni les voir) qu'elle est la femme, et pas l'autre. C, qui agit comme interrogateurévaluateur, peut poser des questions aux deux personnes. Par exemple: «Quelle est la longueur de vos cheveux?» (Rappelons qu'on est en 1950...) La femme pourra toujours dire qu'elle a les cheveux longs, mais l'homme pourra en dire tout autant. Le jeu de l'imitation de Turing implique donc la possibilité du parjure.

Maintenant, imaginons que l'on remplace la personne A par une machine capable de formuler des énoncés (ce qu'on appelle aujourd'hui une "intelligence artificielle»). Turing prédisait qu'aux alentours de l'an 2000, les machines sauraient tromper les interrogateurs au moins $30 \%$ du temps. Si cela advient, alors la question « Est-ce que les machines peuvent penser? » ne se posera même plus, tellement cela ira de soi. Je n'entre pas dans les détails du test ni même dans ses conditions de réussite ${ }^{23}$, car je le considère comme l'introduction à un problème plus général : celui de savoir comment se constitue la différence entre la pensée machinique et la pensée humaine. Dans son article, Turing relève avec beaucoup d'ironie quelques oppositions possibles à sa conception de l'espritmachine :

The «skin of an onion » analogy is also helpful. In considering the functions of the mind or the brain, we find certain operations which we can explain in purely mechanical terms. This, we say, does not correspond to the real mind : it is a sort of skin which we must strip off if we are to find the real mind. But then in what remains, we find a further skin to be stripped off, and so on. Proceeding in this way, do we ever come to the « real » mind, or do we eventually come to the skin which has nothing in it? In the latter case, the whole mind is mechanical ${ }^{24}$.

Turing ne veut pas démontrer la grandeur des capacités «intellectuelles» futures des machines (l'ordinateur, l'intelligence artificielle), comme on le croit trop souvent ; il veut montrer l'indigence de notre propre pensée. Au fond de notre esprit, lorsqu'on y a retiré toutes les opérations qui fonctionnent par binarité, les "mécanismes" comme les différentes couches des pelures d'un oignon, on se retrouve devant « rien ». Le cœur de 
l'oignon est ce rien, et c'est ce rien qui constitue (tout en ne le « constituant » pas, car il n'y a rien à constituer), l'esprit humain.

Plus généralement, on pourrait schématiser cette conception du langage comme suit : la version faible dirait qu'une «vie intérieure » de l'esprit existe, mais que, justement, elle n'est qu'intérieure, inaccessible aux autres ${ }^{25}$. La version forte de cette thèse pourrait toutefois se formuler ainsi : il n'y a pas d'intérieur à l'esprit, puisqu'il n'est qu'un effet du langage, extérieur et superficiel, un ensemble de répétitions d'unités limitées, recombinables infiniment en théorie, mais de manière assez prévisible en pratique (calculable statistiquement, par algorithmes). C'est une fois admise cette indistinction entre les machines et les humains qu'une véritable réflexion sur la Machine pensante est possible, et Turing introduit à la fin de son article une réflexion originale sur l'improgrammabilité de la machine. Ce qu'il appelle les «learning machines» sont des ordinateurs qui incluront en eux-mêmes un élément aléatoire (random element) à partir duquel ils pourront "apprendre ». Cette part improgrammable, c'est justement le lieu d'une certaine « humanité » de la machine, et le lieu de tous les dangers. De 2001: A Space Odyssey à Blade Runner en passant par Star Trek: The Motion Picture, la machinalité de l'intelligence artificielle devient un problème pour l'humanité au moment où elle cesse d'être ce qu'on la force à être: une mécanique. Or, il me semble que la série Battlestar Galactica, particulièrement dans son épisode final, permet de réévaluer les rapports entre la Machine et l'Humanité.

\section{De la machine humaine à l'Homme-machine}

Dans sa manière d'aborder le problème de la machine, Battlestar Galactica se distingue des fictions ayant abordé la possibilité d'une révolte des créatures contre leurs créateurs. Un peu à la manière d'Alan Turing, il s'agit de retrouver le cœur de ce qui distingue l'Homme et la Machine. La fiction à cet égard a toujours servi de révélateur des peurs associées à la robotique comme imitation plus ou moins parfaite de l'humanité. Quelle est la nature de cette imperfection? La réponse à cette question semble être l'originalité de Battlestar Galactica.

Située dans ce qu'on comprend d'abord comme un futur, la série se donne comme une réflexion sur les mythes ou les grands archétypes de notre culture, avec pour grand schème une mythologie partiellement prise des Grecs, des religions abrahamiques, et même de la philosophie hindoue ${ }^{26}$. La série commence en pleine guerre entre les humains et les Cylons, des robots créés par les humains qui se sont révoltés quarante ans auparavant. Alors que la guerre s'était terminée, elle reprend soudainement avec une attaque fulgurante qui réduira l'humanité à une quarantaine de milliers d'âmes.

Pendant les quarante ans séparant la révolte des Cylons et la nouvelle attaque sur laquelle la série débute, les robots se sont développés eux-mêmes jusqu'à prendre une forme humaine, y compris au niveau organique, puisqu'ils ont même développé un code génétique. Alors que les humains se retrouvent en fuite, démunis après la quasidestruction de leur civilisation, on apprend dans la première saison que les Cylons et les humains peuvent se reproduire grâce à l'ADN développé par les Cylons. Pour ce qui est de leur culture, la différence entre les Cylons et les humains pourrait se résumer à un début de monothéisme chez ces premiers, alors que les seconds demeurent polythéistes. L'objectif des humains dans leur fuite est de retrouver une planète mythique appelée la 
Terre, une nouvelle Kobol originelle, la planète mythique. Dans un des derniers épisodes de la quatrième et dernière saison (S04E13, "Sometimes a Great Notion", diffusée le 16 janvier 2009), les humains retrouvent la Terre promise, mais ils la retrouvent complètement dévastée par une guerre nucléaire qui a eu lieu deux mille ans auparavant. Les spectateurs sont amenés à imaginer que cette Terre est la nôtre, détruite par les bombes atomiques.

L'enjeu de la dernière saison qui m'intéressera ici est celui de la survie des deux espèces. Une enfant, née d'une Cylon et d'un humain, est enlevée par les Cylons. Elle serait, dit-on, la clé pour la survie des deux civilisations. Dans un schéma classique de type messianique, l'enfant incarne la possibilité d'une nouvelle naissance pour les deux espèces: alors que les Cylons veulent la disséquer pour l'analyser (mécaniquement, machinalement), les humains la récupéreront et tenteront un accord pour terminer la guerre. En échange de la fille, les humains accorderont le secret de la résurrection aux Cylons, secret gardé par cinq proto-Cylons, chacun possédant une part du secret. Une fois réunies, les différentes parties permettront de découvrir le secret de la résurrection, et donc de la vie éternelle. Or, au dernier moment, le pacte n'ayant pas lieu, la résurrection ne pourra plus se faire mécaniquement, mais par génération, naissance et mort ${ }^{27}$. Un des personnages phares de la série, le Docteur Gaius Baltar, à la fois le traître initial (il est celui qui donne, par amour pour une Cylon, le code qui permet aux Cylons de détruire les colonies), scientifique et (faux) prophète, charlatan et créateur d'une secte, hostile à la démocratie et, finalement, l'instigateur d'un accord avec les Cylons, énoncera, dans un dialogue avec Brother Cavil (un Cylon), l'improgrammabilité de l'à-venir par le cycle, pour les hommes et pour les machines :

BROTHER CAVIL. If that were true, and that's a big 'if', how do I know this force has our best interests in mind? How do you know that God is on your side, Doctor? DOCTOR GAIUS BALTAR. I don't. God's not on anyone side. God is a force of nature, beyond good and evil. Good and evil, we created those. You want to break the cycle? Break the cycle of birth? Death? Rebirth? Destruction? Escape? Death? Well, that's in our hands, in our hands only. It requires that we live in hope, not fear.

L'accord n'ayant pas pu aboutir - le téléchargement du secret de la résurrection n'ayant pas pu être complété - la destruction des Cylons et de l'humanité devient inévitable. L'équipage doit alors se risquer à un dernier "saut » dans l'espace, le dernier, car les vaisseaux spatiaux sont déjà trop endommagés. Les coordonnées du lieu où pourra se rendre l'humanité sont données par un autre personnage essentiel, Kara Thrace, pilote hors-pair, dont on a appris, au cours de cette dernière saison, qu'elle est elle-même un "double ", une « imitation » d'elle-même. Les coordonnées lui sont suggérées par une mélodie qui l'obsède depuis l'enfance. Les coordonnées numériques correspondant aux notes de cette mélodie permettront le transport de l'équipage et le groupe d'humains encore en vie vers la destination finale. Ce dernier lieu, - dernier lieu de l'humanité -, est une planète habitable, peuplée d'hominidés proto-sapiens qui ne possèdent pas le langage, mais donnent une sépulture à leurs morts. Le spectateur de la série comprend alors que l'histoire ne se déroulait pas dans notre futur - comme il est habituel en science-fiction -, mais dans notre passé : la guerre épique entre les Cylons et les humains était depuis le début notre histoire oubliée ${ }^{28}$. Ce nœud que représente l'enfant Héra, progéniture hybride d'un homme et d'une Cylon, est ce qui permet de continuer le cycle. Baltar énoncera alors à nouveau une sentence répétée plusieurs fois au courant de la série, provenant d'un texte sacré : «All of this has happened before, and will happen again » («Tout cela est déjà arrivé, et arrivera encore à nouveau ${ }^{29}$.) » 

Galactica à une réflexion sur l'humanité. Par prothèse d'origine, je désigne ce qui est extérieur, mais en même temps constitue l'intériorité d'une chose. Cette intériorité pourrait se comprendre comme le langage, ce phénomène humain mystérieux qui inclut à la fois la répétition (les sons sont limités et finissent par se répéter), mais aussi la différence (des énoncés sont sans cesse créés), c'est-à-dire l'itérabilité au cœur de l'humanité. De la division entre l'Homme et la Machine, on passe à une division entre ce préjugé et son contraire ; l'Homme et la Machine s'entr'affectent, s'entre-créent. Si on se décide à diviser Homme et Machine absolument, comme le fait une certaine pensée humaniste contemporaine refusant tout apport de la machine, on perpétue une binarité tout à fait machinale, celle notamment de la dualité cartésienne. Penser « humainement », c'est surmonter cette binarité mécanique en réintroduisant une part mécanique dans la pensée humaine. fois un supplément dans le sens qu'il donne un surplus à l'humain, lui est extérieur, et le supplée comme complément. Or, la robotique est également un supplément au sens où elle remplace l'humain, lui est potentiellement intérieure, et le supplée comme suppléant ${ }^{31}$.

Depuis le développement de la cybernétique, l'image de la Machine a pris le rôle du virus contaminant le «sujet politique " indemne. Battlestar Galactica permet de repenser à nouveau la subjectivité contemporaine. Le sujet toujours-déjà incomplet, entamé - on pourrait aussi dire «abîmé » dès l'origine. L'abîme, ce n'est pas l'abysse (en latin abyssus) dont l'origine étymologique provient d'un mot grec signifiant le «sans fond ». L'origine du latin abismus qui donne abîme est incertaine : il pourrait provenir d'imus, le superlatif d'inferus : plus au fond que le fond ou plus intérieur que l'intérieur ${ }^{32}$. On trouve ici à la fois intériorité et répétition : l'union de l'intériorité de la conscience (celle qu'on attribue à l'Homme), et répétition machinale. La machine, par sa répétition, est intérieure à l'Homme. La science-fiction en général et Battlestar Galactica en particulier - comme série télévisuelle dont la forme esthétique est fondée sur la répétition hebdomadaire du récit 
en continu - permettent de remettre en question des sédimentations philosophiques souvent répétées machinalement, et celle, notamment, de l'idéal philosophique d'un sujet humain autonome.

\section{BIBLIOGRAPHIE}

ACHOUCHE Mehdi, « De Babylon à Galactica : la nouvelle science-fiction télévisuelle et l'effetréalité », TV/Series, n 1, éd. Ariane Hudelet et Sophie Vasset, juin 2012, p. 313-330. URL : http:// tvseries.revues.org/1515

ACHOUCHE Mehdi, «Le "reboot”, hyper-remake contemporain », Représentations, Revue électronique du CEMRA, décembre 2015, p. 20-41, http://representations.u-grenoble3.fr/ spip.php?article37

ARP Robert, et Tracie MAHAFfeY, « “And They Have A Plan” : Cylons As Persons », Battlestar Galactica and Philosophy: Knowledge Here Begins Out There, éd. Jason T. Eberl, Malden, Blackwell Publishing, 2008, p. 55-63.

BATAILLE Sylvaine, «Les pièges du temps : la réappropriation de l'Antiquité gréco-latine dans Battlestar Galactica (Sci Fi, 2003-2009) », GRAAT, $\mathrm{n}^{\circ}$ 6, éd. Sarah Hatchuel et Monica Michlin, décembre 2009, p. 86-106. URL : http://www.graat.fr/backissuepiegesseriestv.htm Boudou Nadine, «Les imaginaires cinématographiques de la menace », Sociétés, n 123, 2014/1, p. 117-126.

BRADLEY James, «All of this has happened before and will happen again : Humanity, inhumanity and otherness in Battlestar Galactica ", d'abord publié dans Meanjin, vol 67, n 4, 2008. Repris dans City of tongues. Blogue, http://cityoftongues.com/non-fiction/all-of-this-has-happened-before EBERL Jason T. (éd.), Battlestar Galactica and Philosophy: Knowledge Here Begins Out There, Malden, Blackwell Publishing, 2008.

DERRIDA Jacques, De la grammatologie, Paris, Éditions Gallimard, 1967.

DERRIDA Jacques, Le Monolinguisme de l'autre, ou la prothèse d'origine, Paris, Éditions Galilée, 1996. DERRIDA Jacques, Papier Machine. Le ruban de machine à écrire et autres réponses, Paris, Éditions Galilée, 2001.

DESCARTES René, Discours de la méthode, éd. Victor Cousin, Éditions Levrault, 1824.

DICK Philip K., Do Androids Dream of Electric Sheep ?, Doubleday, 1968. Trad. Serge Quadruppani, Les androïdes rêvent-ils de moutons électriques ?, Paris, Champ Libre, 1976.

FREITAG Michel, « Postmodernité de l'Amérique », avant-propos de René Lemieux, Les Cahiers de l'idiotie, vol. 1, n 1, 2008, p. 69-119.

GIROUX Dalie, « Le corps et les signes : sur la relation entre le langage européen et l'espace américain », Les Cahiers de l'idiotie, vol. 1, n 1, 2008, p. 121-147. 
HAWKING Stephen, «Stephen Hawking warns artificial intelligence could end mankind », entretien avec Rory Cellan-Jones, BBC, 2 décembre 2014, http://www.bbc.com/news/ technology-30290540

HOWIE Luke J., Terror on the Screen. Witnesses and the Re-animation of 9/11 as Image-event, Popular Culture and Pornography, New Academia Publishing, 2010.

HowIE Luke J., « They Were Created by Man... and They Have a Plan : Subjective and Objective Violence in Battlestar Galactica and the War on Terror ", International Journal of Žižek Studies, vol. 5, $\mathrm{n}^{\mathrm{o}} 2,2011, \mathrm{np}$.

JOHNSON David Kyle, " "A Story That Is Told Again, and Again, and Again" : Recurrence, Providence, and Freedom », in Battlestar Galactica and Philosophy : Knowledge Here Begins Out There, éd. Jason T. Eberl, Malden, Blackwell Publishing, 2008.

JOHNSON-LEWIS Erika, " Torture, Terrorism, and Other Aspects of Human Nature », in Cylons in America : Critical Studies of Battlestar Galactica, éd. C. W. Marshall et Tiffany Potter, Continuum Publishing, 2008, p. 27-39.

JOWETT Lorna, « Frak Me : Reproduction, Gender, Sexuality », in Battlestar Galactica : Investigating Flesh, Spirit and Steel, éd. Roz Kaveney et Jennifer Stoy, Londres, I. B. Tauris, 2010, p. 59-80.

KAVENEY Roz et Jennifer STOY (éd.), Battlestar Galactica : Investigating Flesh, Spirit and Steel, Londres, I. B. Tauris, 2010.

DE LA METTRIE, Julien OFFRAY, L'Homme-Machine, précédé de Lire La Mettrie. P. L. Assoun (éd.), Paris, Éditions Gallimard, 1999.

LEMIEUX René, « La traduction à l'époque de sa reproductibilité technique : l'im-possible dissonance interculturelle », dans Actes du colloque étudiant du CELAT : « Les dissonances du vivreensemble », éd. Eleonora Diamanti, Mariza Rosales, Dagmara Zawadzka, printemps 2014, p. 19-30. LEVINE Joseph, « Materialism and qualia : the explanatory gap », Pacific Philosophical Quarterly, $\mathrm{n}^{\circ}$ 64, p. 354-361.

MARSHALL C. W., et Tiffany POTTER, « "I See the Patterns" : Battlestar Galactica and the Things That Matter », Cylons in America : Critical Studies of Battlestar Galactica, éd. C. W. Marshall et Tiffany Potter, Continuum Publishing, 2008, p. 1-10.

MORI Masahiro, « Bukimi no tani [The uncanny valley] », Energy, vol. 7, nº 4, p. 33-35.

RYMAN Geoff, « Adama and (Mitochondrial) Eve : A Foundation Myth for White Folks », Battlestar Galactica : Investigating Flesh, Spirit and Steel, éd. Roz Kaveney et Jennifer Stoy, Londres, I. B. Tauris, 2010, 37-58.

TURING Alan, « Computing Machinery and Intelligence », Mind, vol. 59, $\mathrm{n}^{\circ}$ 236, octobre 1950, p. $433-460$.

\section{NOTES}

1. Mehdi Achouche, «Le "reboot", hyper-remake contemporain » (Représentations, décembre 2015), p. 22.

2. Ibid.

3. Voir Michel Freitag, «Postmodernité de l'Amérique ", Les Cahiers de l'idiotie, vol. 1, no 1, 2008, p. 88. On pourra aussi consulter, pour une réflexion sur la fondation américaine, Dalie Giroux 
pour qui « la fondation se trouve en écho de toute itération culturelle de type politique, et que c'est par là qu'il est possible de caractériser quelque chose comme une pensée proprement American (sic) ». Dalie Giroux, « Le corps et les signes : sur la relation entre le langage européen et l'espace américain ", Les Cahiers de l'idiotie, vol. 1, n 1, 2008, p. 123.

4. Sur cette question, on pourra lire Goeff Ryman, "Adama and (Mitochondrial) Eve: A Foundation Myth for White Folks ", dans Roz Kaveney et Jennifer Stoy (éd.), Battlestar Galactica : Investigating Flesh, Spirit and Steel, Londres, I. B. Tauris, 2010. Sans vouloir minimiser le « racisme structurel » de la série, je pense qu'il est possible de développer une réflexion à partir d'elle qui peut aussi permettre, en fin de compte, de comprendre les points aveugles de notre société. Ainsi, comme le propose Sylvaine Bataille, je prends les aspects « américains » de la série (l'usage d'une seule langue, l'anglais, le système politique présenté, une démocratie parlementaire, les mythes judéo-chrétiens et gréco-romains racontés, etc.) comme des éléments de "réalisme». Voir Sylvaine Bataille, «Les pièges du temps : la réappropriation de l'Antiquité gréco-latine dans Battlestar Galactica (Sci Fi, 2003-2009) », «Les Pièges des nouvelles séries télévisées » (éd. Sarah Hatchuel et Monica Michlin), Revue du GRAAT n 6, décembre 2009, p. 86-106.

5. J'emprunte l'expression "prothèse d'origine " à Jacques Derrida dans Le Monolinguisme de l'autre (il s'agit du sous-titre: Ou la prothèse d'origine, Paris, Éditions Galilée, 1996). Derrida ne réutilisera pas l'expression dans le livre, je me permets ainsi de lui donner un nouvel usage.

6. À ce propos, on pourra consulter Mehdi Achouche, «De Babylon à Galactica: la nouvelle science-fiction télévisuelle et l'effet-réalité », TV/Series, $n^{\circ} 1$, juin 2012, p. 313-330, ainsi que Luke J. Howie, «They Were Created by Man... and They Have a Plan : Subjective and Objective Violence in Battlestar Galactica and the War on Terror ", International Journal of Žižek Studies, vol. 5, $\mathrm{n}^{\circ} 2,2011, \mathrm{np}$. Voir aussi Luke J. Howie, Terror on the Screen. Witnesses and the Re-animation of 9/11 as Image-event, Popular Culture and Pornography, New Academia Publishing, 2010, ainsi que Erika Johnson-Lewis, "Torture, Terrorism, and Other Aspects of Human Nature ", Cylons in America: Critical Studies of Battlestar Galactica, éd. C.W. Marshall et Tiffany Potter, Continuum Publishing, 2008, p. 27-39.

7. Sur l'imaginaire de la catastrophe au cinéma et à la télévision, voir Nadine Boudou, «Les imaginaires cinématographiques de la menace ", Sociétés, $n^{\circ}$ 123, 2014/1, p. 117-126.

8. Selon lui, le développement de l'intelligence artificielle pourrait représenter la fin de l'espèce humaine : «Humans, who are limited by slow biological evolution, couldn't compete and would be superseded. » («Stephen Hawking warns artificial intelligence could end mankind », Entretien avec Rory Cellan-Jones, BBC, 2 décembre 2014.) Il avait auparavant affirmé en 2010 qu'il fallait cesser de tenter de contacter de possibles intelligences extraterrestres avec le programme SETI de la NASA au risque que ces extraterrestres puissent atteindre la Terre. Ce contact serait l'équivalent, selon lui, de l'arrivée de Colomb en Amérique.

9. René Descartes, Discours de la méthode, cinquième partie, éd. Victor Cousin, Éditions Levrault, tome I, 1824, p. 185-186.

10. Jacques Derrida, «L'avant-dernier mot: archives de l'aveu ", Papier Machine. Le ruban de machine à écrire et autres réponses, Paris, Éditions Galilée, 2001, p. 35. Pour une mobilisation de ce texte dans une argumentation reliée au langage, en particulier au sujet de la traduction, voir René Lemieux, «La traduction à l'époque de sa reproductibilité technique: l'im-possible dissonance interculturelle », Actes du colloque étudiant du CELAT: «Les dissonances du vivreensemble », éd. Eleonora Diamanti, Mariza Rosales, Dagmara Zawadzka, printemps 2014, p.19-30. 11. Qu'on associe parfois à IBM (dont chaque lettre est la suivante dans l'ordre alphabétique).

12. En ce qui a trait à l'imitation quasi parfaite, on peut rappeler que dans les années 1970, l'informaticien Masahiro Mori avait théorisé le niveau d'acceptation qu'auraient les humains envers les robots en supposant que l'acceptation serait proportionnelle à la ressemblance entre les machines et les hommes, sauf pour un moment très proche de la ressemblance parfaite où, à ce moment, au contraire, on assisterait à un rejet total de l'apparence des robots. Ce rejet a été 
appelé la «vallée de l'étrange », en anglais «the uncanny valley» (traduction du japonais bukimi, " ce qui tient du mauvais présage, ce qui est menaçant », mais surtout de l'allemand unheimlich chez Sigmund Freud « l'inquiétante étrangeté »). La courbe ascendante de l'acceptation chuterait rapidement pour ne remonter que lorsque la distinction esthétique entre homme et machine ne serait plus possible. Cette hypothèse tiendrait de la répulsion instinctive devant un cadavre, un mort ou une personne gravement malade : toute image « abjecte » du soi humain. L'hypothèse a aussi été proposée pour l'intelligence artificielle : une intelligence artificielle qui, par exemple, laisserait penser qu'elle ressent des émotions ou de la douleur, mais sans l'exprimer entièrement comme on s'imagine qu'un humain les ressent, ferait naître ce sentiment d'inquiétante étrangeté.

13. Star Trek: The Motion Picture, réal. Robert Wise, avec William Shatner, Leonard Nimoy, DeForest Kelly, Persis Khambatta et Stephen Collins.

14. Après le programme Pioneer (19 sondes langées de 1958 à 1978), la NASA lance les deux seules sondes dans le cadre du programme Voyager en 1977.

15. En termes juridiques, on pourrait parler de « personne » qui ne signifie pas un individu, mais un titulaire de droits et d'obligations. Selon les époques, on n'a pas toujours reconnu aux hommes de certaines races, aux femmes, aux enfants ou aux animaux une personnalité juridique. Pour une discussion philosophique sur cette question en rapport à Battlestar Galactica, on pourra lire Robert Arp et Tracie Mahaffey, "And They Have A Plan": Cylons As Persons ", dans Battlestar Galactica and Philosophy: Knowledge Here Begins Out There, éd. Jason T. Eberl, Malden, Blackwell Publishing, 2008, p. 55-63.

16. Un trope encore une fois surexploité dans le cadre de la science-fiction, et particulièrement dans Star Trek avec le personnage de Data dans The Next Generation, est celui du "robot " ne saisissant pas l'humour au "second degré », la situation devenant elle-même un ressort comique des relations entre les êtres vivants et les machines. Auparavant, c'était une espèce extraterrestre (les Vulcains comme Spock) qui prenait ce rôle de « logicien » sans émotion.

17. La seule option possible est alors la «complémentarité » : à la fin du film, on assistera à l'union mystique, dans l'amour, entre la représentation corporelle de V'Ger en femme et l'ancien amant de celle-ci (alors qu'elle était toujours un être vivant): cette union résultera dans l'annihilation des deux entités au profit de la survie de la Terre.

18. Philip K. Dick, Do Androids Dream of Electric Sheep ?, publié en 1968, réédité après l'adaptation du film sous le titre Blade Runner. Traduit en français et publié en 1976 aux éditions Champ Libre.

19. C'était le cas de 2001: A Space Odyssey mentionné plus haut, mais aussi du film Terminator (1984) et sa suite Terminator 2: Judgment Day (1991), réalisés par James Cameron, avec Arnold Schwarzenegger et Linda Hamilton. Dans ces deux films, une intelligence artificielle contrôlant le système de défense des États-Unis, Skynet, est mise en fonction le 12 août 1997 et commence à évoluer à un rythme exponentiel, la conduisant à accéder à une conscience d'elle-même (selfawareness) le 29 août 1997, ce qui la mènera à déclarer la guerre à l'humanité en lançant des obus nucléaires sur la Russie.

20. C'est ce que dit le célèbre monologue du réplicant Roy Batty (joué par Rutger Hauer) «Tears in rain ", écrit par Hauer lui-même la veille du tournage de la scène : "I have seen things you people wouldn't believe. Attack ships on fire off the shoulder of Orion. I watched c-beams glitter in the dark near the Tannhäuser Gate. All those moments will be lost in time, like tears in rain. Time to die... »

21. Définir la pensée comme une forme de «machinerie » a eu ses prédécesseurs, le plus célèbre étant peut-être Julien Offray de La Mettrie, auteur de L'Homme-Machine, publié au XVIII ${ }^{\mathrm{E}}$ SIÈCLE. C'EST TOUTEFOIS À TURING QU'ON DOIT LE PREMIER D'INVERSER LA DONNE DANS LA RELATION ENTRE L'HOMME ET LA MACHINE EN PROPOSANT UNE MACHINE QUI PEUT APPRENDRE, COMME JE L'EXPLIQUERAI.

22. Alan Turing, «Computing Machinery and Intelligence », Mind, vol. 59, $\mathrm{n}^{\circ} 236$, octobre 1950, p. 433-460. 
23. Déjà, l'interprétation du test fait polémique: est-ce que l'interrogateur saura qu'il est possible qu'il s'adresse à une machine ? Est-ce vraiment nécessaire d'avoir un « humain » présent comme témoin ou l'alternative humain-machine suffit-elle ? Pour ce qui est de la " réussite » du test, on prétend l'avoir réussi dès les années 1960.

24. Alan M. Turing, ibid., p. 454-455.

25. C'est par exemple le problème des qualia, ces qualités sensibles, mais subjectives qui ne sont jamais ultimement communicables : il n'y a pas de moyen de saisir ce que ressentent d'autres personnes (problème dit du «explanatory gap », voir Joseph Levine, "Materialism and qualia : the explanatory gap », Pacific Philosophical Quarterly, n 64, p. 354-361).

26. Pour plus de détails formels sur cette mythologie, on pourra lire Mehdi Achouche, «De Babylon à Galactica : la nouvelle science-fiction télévisuelle et l'effet-réalité ", TV/Series, $n^{\circ} 1$, juin 2012, p. 313-330, et Sylvaine Bataille, «Les pièges du temps: la réappropriation de l'Antiquité gréco-latine dans Battlestar Galactica (Sci Fi, 2003-2009) », Revue du GRAAT n ${ }^{\circ}$ 6, éd. Sarah Hatchuel et Monica Michlin, déc. 2009, p. 86-106.

27. Pour une réflexion plus longue sur la question de la procréation et sur les enjeux de genre dans la série, on pourra consulter Lorna Jowett, "Frak Me: Reproduction, Gender, Sexuality ", Battlestar Galactica : Investigating Flesh, Spirit and Steel, éd. Roz Kaveney et Jennifer Stoy, Londres, I. B. Tauris, 2010, p. 59-80.

28. Pour Mehdi Achouche, la série Battlestar Galactica n'est « ni située dans le futur ni vraiment dans le passé, malgré les révélations finales de la série », mais plutôt « dans une sorte d'univers parallèle au nôtre » (« De Babylon à Galactica : la nouvelle science-fiction télévisuelle et l'effetréalité », art. cit., p. 320). Sans être "fausse », cette appréciation de la temporalité cyclique développée par la série peut toutefois nous détourner de ce que la série peut apporter de plus important selon moi, à savoir un récit des origines et des finalités de l'« humanité ». Il faut peutêtre simplement imaginer, comme l'énonce la série elle-même, que les aventures racontées dans la série ont déjà plusieurs fois eu lieu, et auront lieu encore à de multiples reprises.

29. L'auteur James Bradley y voit, par sa récurrence dans la série et par la récurrence que la phrase suggère, l'isomorphe sémantique de ce que les Cylons représentent dans la série, l'inauthentique répétition de l'humanité, les Cylons comme simulacres ou doppelgänger, des instances de l'unheimlichkeit freudienne : "The mantra [...] might also be seen as another instance of this Freudian pattern of recurrence, or indeed of that other most uncanny sense of repetition, déjà vu. " James Bradley, "All of this has happened before and will happen again : Humanity, inhumanity and otherness in Battlestar Galactica", City of tongues, blogue, en ligne : <http:// cityoftongues.com/non-fiction/all-of-this-has-happened-before/> Sylvaine Bataille rappelle que la formule provient du dessin animé de Disney, Peter Pan (Sylvaine Bataille, op. cit., p. 95).

30. Paradoxalement, la série se termine sur un épilogue où l'on voit les deux "anges » qui suivent, en les imitant, le couple composé de Baltar et de la Cylon Numéro 6, 150000 ans plus tard, à notre époque, contemplant où nous en sommes sur le plan civilisationnel. On voit sur écran que viennent d'être découverts les restes de "l'Ève mitochondriale ", l'ancêtre matrilinéaire commune de toute l'humanité - Héra, suppose-t-on. On voit défiler sur l'écran mis en abyme différentes images de récents exploits technologiques en robotique. Le dialogue final énonce le danger que se répètent, encore une fois, toutes les catastrophes vécues par l'humanité. On peut voir dans cet avertissement aux spectateurs l'expression d'une mélancolie rousseauiste, comme si les créateurs de la série n'avaient pas réussi à aller au bout de leurs idées ; Geoff Ryman n'a pas tort d'y lire une certaine technophobie. Voir Geoff Ryman, op.cit., p. 55-56.

31. Sur la question du supplément que j'emprunte à Jacques Derrida, voir son livre De la grammatologie, Paris, Éditions Gallimard, 1967.

32. C'est dans ce sens qu'on utilise l'expression « mise en abyme » qui signifiait primairement la répétition d'une figure à l'intérieur d'elle-même. 


\section{RÉSUMÉS}

Cet article se propose d'explorer comment la série Battlestar Galactica de Ronald D. Moore (Sci-Fi, puis Syfy, 2003-2009) n'est pas seulement une série télévisée de science-fiction, mais constitue une critique philosophique forte de la pensée dualiste moderne de René Descartes et de la distinction entre l'Homme (doué d'un langage raisonné) et son Autre (l'animal-machine), qui aujourd'hui encore sous-tendent l'humanisme. Contre Descartes, et avec Derrida, une interprétation nouvelle des origines de la relation entre Cylons (les robots humanoïdes de Battlestar Galactica) et humains est proposée dans les dernières révélations de la série : ce qui est de l'ordre de la machine - de la machinalité, du mécanique ou du répétitif - devient ce qui est nécessaire comme prothèse à la nature pour que l'humanité soit pensable. L'humanité n'est plus l'envers de la machine, mais ce qui se constitue à partir de la machine.

This article discusses the possibility that Ronald D. Moore's Battlestar Galactica (Sci-Fi, then Syfy, 2003-2009) is not merely a science-fiction television series, but a strong philosophical critique of the modern dualistic thinking of René Descartes, in which a distinction between Man (constituted by a rational language) and his Other (the animal-machine) constitutes the basis for humanism. By engaging with the philosopher Jacques Derrida, a new interpretation emerges concerning the origins of the relationship between Cylons (Battlestar Galactica's androids) and humans (one revealed in the last episodes of the show): what can be found on the level of the machine - the mechanical or the repetitive - is a prosthesis to nature that is required in order for humanity to be thinkable. Humanity is thus not the opposite of the machine, but what can arise from the machine.

\section{INDEX}

Mots-clés : Battlestar Galactica, Derrida Jacques, Descartes René, Turing Alan, humanité, machine, cinéma, science-fiction

Keywords : Battlestar Galactica, Derrida Jacques, Descartes René, Turing Alan, humanity, machine, cinema, science fiction

\section{AUTEUR}

\section{RENÉ LEMIEUX}

René Lemieux est politologue de formation et docteur en sémiologie de l'Université du Québec à Montréal. Il est chercheur associé à l'Observatoire du Discours Financier en Traduction de l'Université Concordia (Montréal). Ses recherches portent principalement sur les théories de la traduction et de la réception, ainsi que sur la philosophie française contemporaine.

René Lemieux has a background in political philosophy and holds a PhD in semiology from Université du Québec à Montréal. He is a research fellow at the Observatory on Financial 
Discourse in Translation based in Concordia University (Montréal). His research primarily

focuses on translation and reception theories, as well as on contemporary French philosophy. 